\title{
Feature Extraction for Emotion Recognition in Speech with Machine Learning Algorithm
}

\author{
R. Aishwarya ${ }^{1}$, Yogitha. $\mathbf{R}^{2}$, M. Selvi ${ }^{3}$, G. Kalaiarasi ${ }^{4}$ \\ ${ }^{1}$ Sathyabama Institute of Science and Technology, India, aishwarya.cse@ sathyabama.ac.in \\ ${ }^{2}$ Sathyabama Institute of Science and Technology, India, yogitha.cse@ sathyabama.ac.in \\ ${ }^{3}$ Sathyabama Institute of Science and Technology, India, selvi.cse@ sathyabama.ac.in \\ ${ }^{4}$ Sathyabama Institute of Science and Technology, India, kalaiarasi.cse@ sathyabama.ac.in
}

\begin{abstract}
Feelings acknowledgments from Debate are among the most common significant sub spaces in the field of sign handling. Right now, framework is a two-organize approach, to be specific element extraction and grouping motor. Investigation of first and second request contrasts of amicability highlights assumes a significant Its job in passionate energy about words. Expanding on this, we propose another model utilizing perceptual substance quality of voice and request Acceptance of passionate language varieties for speakers impartial. Proof from tests shows that arranged Fourier parameter (FP) highlights are viable in recognizing different passionate states in discourse signals. This improves the acknowledgment over the strategies Use of the cepstral force work Mel (MFCC) highlights. Specifically, when consolidating FP with MFCC, PLP and LPC the acknowledgment rates can be additionally improved. After that the KNN classifier is utilized to order the right class of the feeling signal.
\end{abstract}

Key words: Tendency to feel recognition, MFCC, SVM, Dark Vector Model Support, Specific Motor-Encoder, Loaded Motor-Encoder.

\section{INTRODUCTION}

Identification of feelings in speech is a enormously latest Spoken language testing subject processing since it has been studied at some point of best the previous years. it has received quite a few interests, now not only inside the educational field but additionally within the industry, thanks to the multiplied Unit performance and consistency. Appreciation of Language Impulses can be used in numerous applications. These consist of psychiatric analysis, clever toys, lie detection, shrewd name center, educational software program, and so on. Most of the research take advantage of the pre- segmented sequences of a unmarried speaker and not the spontaneous verbal exchange between a couple of audio system. This method makes the paintingstoughto generalize for the records gathered in a natural way. Others analyst used different algorithms for the popularity of human emotions from speech. The automobile-encoder (AE) is usually followed for developing a deep shape to inspire learning of structural functions. Yu et al. Proposed a graphic regularized self-coder, aiming to undertake a graph to manual encoding and encoding.

\section{RELATED WORK}

Numerous analysts have executed different discourse feeling acknowledgment models utilizing various arrangements of highlights. Luger and Yang applied the Berlin Emotional Expression Database to characterize six emotions (outrage, satisfaction, misery, fatigue, dread, and impartial) with a Bayesian classifier displayed with Gaussians utilizing prosodic highlights and voice high-quality. Schuller et al. Ordered six wonderful feelings depending on a mix of a Gaussian Mixture Model (GMM) and a hid Markov Model (HMM) technique utilizing both articulated enthusiastic talks and actual elements in English and German and making use of pitch and vitalityfeatures shows in figure 1 .

Schuller and $\mathrm{Al}$ have completed past due tackle the impact of racket and enhancer conditions on unique and unconstrained records. The talk covered two databases, the Danish Emotional Speech Corpus and the Berlin Emotional Speech Database, and the unconstrained talk commenced from a German corpus of information of young people speaking with a human managed robot. They used selection bushes to bunch feeling situation to 2 specific guides of motion of functions, essentialness and absurd traits, combined mass effective (MFCC) coefficients, and extrafeatures. K.V.Krishna and P.Krishna used the SAVEE database, which are going to use bunch of feelings concern to MFCC traits and Subband-based totally Parameter (SBC) approach. These features are described using the Gaussian Mixture Model (GMM) SBC methodology achieve with in $70 \%$ confirmation as opposed to $51 \%$ affirmation with the MFCC estimation. 


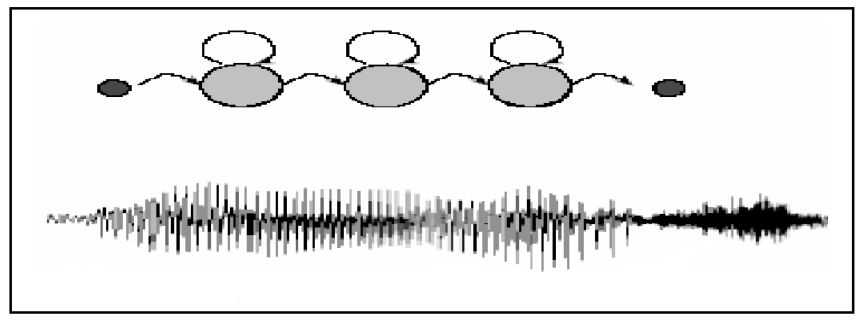

Figure 1: GMM and HMM Model

Generally, ASR frameworks use Mel-recurrence cepstral coefficients (MFCCs) as the acoustic perception. These perform very well in clean coordinated conditions and have been utilized in a few bests in class ASR frameworks. Lamentably, MFCCs are helpless to commotion and their exhibition debases significantly with increments in clamor levels and channel corruptions. To represent MFCC's helplessness to commotion and channel debasements, scientists have effectively looked to get a strong acoustic list of capabilities. Research on clamor vigorous acoustic highlights ordinarily means to produce commotion remunerated highlights for the acoustic-model preparing and such highlights can be created in two different ways: utilizing discourse upgrade based methodologies, where the uproarious discourse signal is improved by decreasing commotion defilement followed by cepstral include extraction; or by utilizing clamor hearty discourse handling draws near, where commotion strong changes.[1]. A significant trouble of discourse handling lives in the way that different useful components are mixed together, thus at whatever point we decipher for a specific factor, every single other factor contribute as vulnerabilities.

An instinctive plan to manage the data mixing is to factorize the discourse signal into individual educational elements at the edge level. In any case, it ends up being exceptionally troublesome, due to at any rate two reasons: Firstly, how these components are blended is hazy and appears to be profoundly intricate; Secondly, and maybe more in a general sense, some central point, especially the speaker quality, carries on as long haul distributional properties instead of brief timeframe designs. It has been halfway exhibited by the way that a large portion of the effective speaker acknowledgment draws near. Therefore, there is a wide doubt that discourse signals are brief timeframe factorizable[2].

Our investigation manages the examination of the NonLinear Spectral Subtraction (NSS) technique with the SVD-based commotion expulsion plot so as to improve debilitated discourse preceding feed it to the discourse acknowledgment framework. The SVD-based strategy of a sign depends on the eigen- investigation of its covariance network. The sign can be viewed as a vector of measurement $\mathrm{N}$ and it is anticipated onto the solitary vectors of a $\mathrm{p} \times \mathrm{N}$ Hankel grid, where $\mathrm{p}$ is the model request viable. This activity is comparable to versatile sifting the sign with a channel whose recurrence reaction is impacted by the otherworldly substance of the sign [3].

In our far-off discourse assortment task, we utilized three discourse datasets and a commotion dataset as the source information. These discourse datasets are recorded in the soundproof room or calm office. In this discourse information, around 1-second-long quietness was added to the two parts of the bargains. The general length of the source discourse information is around 82 hours. The clamor dataset comprises of twelve sorts of lounge room commotion, for example, TV, fridge, forced air system, vacuum cleaner, cell phone rings, music, foundation discussion, and so forth., every one of which is 5-10 moment long. The insights regarding this source information are recorded in Table I. The call words and sentences in Speech dataset I allude to the three words and twelve sentences picked for calling and ordering the conversational robot in which our ASR framework will be installed.[4,6]. The Liquid State Machine (LSM) is another as of late proposed technique that works legitimately on crude information. The LSM depends on a system of spiking neurons that are a lot nearer to organic neurons than the rate-based model utilized in CNNs and DNNs. In spite of its hypothetical intrigue, the LSM is delayed in finding reasonable applications. The primary issue while executing a LS is to make a particular supply structure that is best adjusted to the job needing to be done [7].

Right now, issue is illuminated by presenting earlier information about the human discourse creation framework into the LSM. With no misfortune as far as data, the discourse signal is isolated into two segments: the source and the vocal tract. Exclusively, every part is simpler to process by a store of spiking neurons. Moreover, the consideration of a creation model in the acknowledgment framework is advocated by the engine hypothesis of discourse recognition, that expresses that individuals see discourse by distinguishing the vocal tract signals that deliveredit.[5,8]. Around most of the event feeling talk affirmation is done by expelling Mel frequency cepstral coefficient (MFCC) features followed by applying it to classifier. All signals were first isolated by a pass channel with pre-highlight coefficient 0.97 . The first $3 \mathrm{MFCC}$ and related delta-and twofold delta MFCC swore expelled to shape a 39-dimensional component vector. It means, generally outrageous, least, center and standard deviation were moreover decided out, which incited a 195-dimensional MFCC incorporate vector through and through. These features are then applied to classifier for request. Gives lower affirmation rate. Use cepstral examination shown in figure 2. 


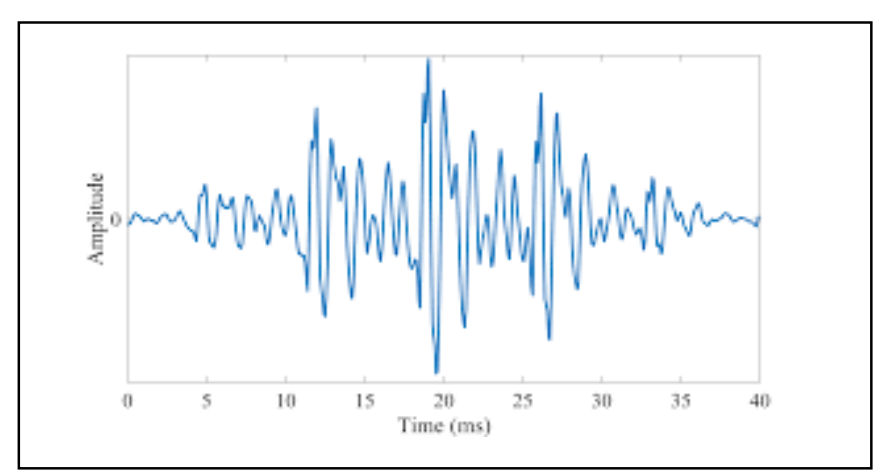

Figure 2: Ceptsral Examination

\section{PROPOSED METHODOLOGY}

At this point in time, propose a ton of symphonious courses of action, name after Four parameter features, for perceive perceptual substance for voice quality features other than conventional ones. A very few new FP's will be surveyed on talk databases. It is one of the values undergoing to apply another course of action of FP's explicitly, with $1^{\text {st }}$-and second- demand contrasts without speaker talk feeling affirmation. Peruse an information voice signal into the mat lab. Add an information voice to the workspace, utilizing the mired order. In picture handling or sign preparing, it is characterized as the activity of recovering an info voice signal from some source, typically an equipment-based hotspot for handling. It is the initial phase in the work process grouping in light of the fact that, without an info voice signal, no handling is conceivable. The voice signal that is gained is totally natural shows in Figure 3.

\subsection{Feature Extraction}

In AI, design acknowledgment and in photograph managing or voice sign getting ready spotlight extraction starts from an underlying association of predicted records and assembles inferred values (highlights) anticipated to an instructive and non- excess, encouraging the results studying of hypothesis steps, and instances prompts a better understanding.

\section{INPUT SPEECH}

FEATURE EXTRACTION

FEATURE NORMALIZATIO N

KNN MACHINE CLASSIFICATIO N

Figure 3: System Architecture
On an point while information to calculation is simply to substantial ever be prepared and it's miles Suspected for repetitive (as an instance a comparable estimation within toes and meter, Or redundancy of pics exhibited in pixel), that point has a tendency to be modified into a decreased arrangements of highlights(named after a component vector). Deciding subset of undergoing highlights known as consist of desire. The highlights are re-lied up-on to incorporate the essential records from information, with a goal that appropriate project may carried out via making use of this faded portrayal in preference to the entire introductory data. Here we use mfcc, plp and lpcfor encompass extraction.

\subsection{Feature normalization}

Standardization is a method regularly applied as a major aspect of information groundwork for AI. The objective of standardization is to change the estimations of numeric sections in the dataset to a typical scale, without mutilating contrasts in the scopes of qualities. For AI, each dataset doesn't require standardization. For instance, consider an informational collection containing two highlights, age(x1), and income(x2). Where age ranges from $0-100$, while salary ranges from 0-20,000 and higher. Pay is around multiple times bigger than age and ranges from 20,000-500,000. In this way, these two highlights are in altogether different extents. At the point when we do encourage investigation, as multivariate direct relapse, for instance, the ascribed salary will naturally impact the outcome progressively because of its bigger worth. In any case, this doesn't really mean it is increasingly significant as an indicator.

\subsection{Classification}

Voice test grouping alludes to the undertaking of extricating data classes from a multiband voice signal. The subsequent raster from picture (or) voice signal arrangement can be utilized to make topical maps. The prescribed method to perform arrangement and multivariate examination is through the Image or voice test Classification toolbar. There are numerous grouping calculations are accessible and some order calculation that are given underneath, A) KNN (K-NEAREST Neighbor).

System Configuration is the hypothetical model that describes the structure, direct and more points of view on a structure. A designing delineation is a traditional depiction and depiction of a system, sifted through with the end goal that supports considering the structures and practices of the system. A system designing can involve structure parts and the sub systems developed, that will participate to realize the general system. There have been tries to formalize lingos to depict structure building; everything considered these are called plan delineation vernaculars.

\section{EXPERIMENTAL RESULTS}

Talk feeling affirmation, which is portrayed as expelling the enthusiastic states of a speaker from their talk, is pulling in more thought. It is acknowledged that talk feeling affirmation can improve the introduction of talk affirmation 
structures and is hence observation and area of perhaps unsafe events and social protection systems. Talk feeling affirmation is particularly useful in manmachine correspondence. To reasonably see emotions from talk hails, the trademark features must be expelled from harsh talk information and changed into fitting plans that are proper for extra taking care of. Investigators have played out various assessments. highlights from passionate discourse flags and approved it on notable database. It is seen that various feelings led to various highlights. Moreover, various highlights were assessed for speaker-free feeling acknowledgment by utilizing KNN classifier. The examination demonstrated that various highlights are successful in portraying and perceiving feelings in discourse signals. Also, it is conceivable to improve the presentation of feeling

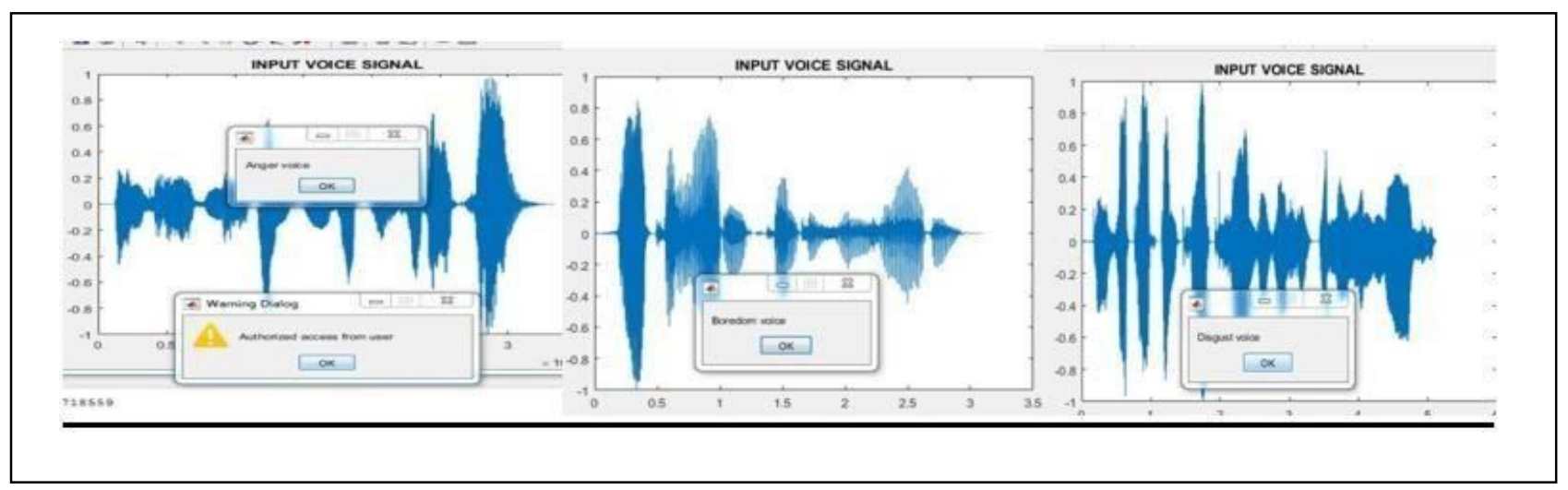

Figure 4: Speech Emotion Recognition using MFCC (Anger, Boredom, Disgust)

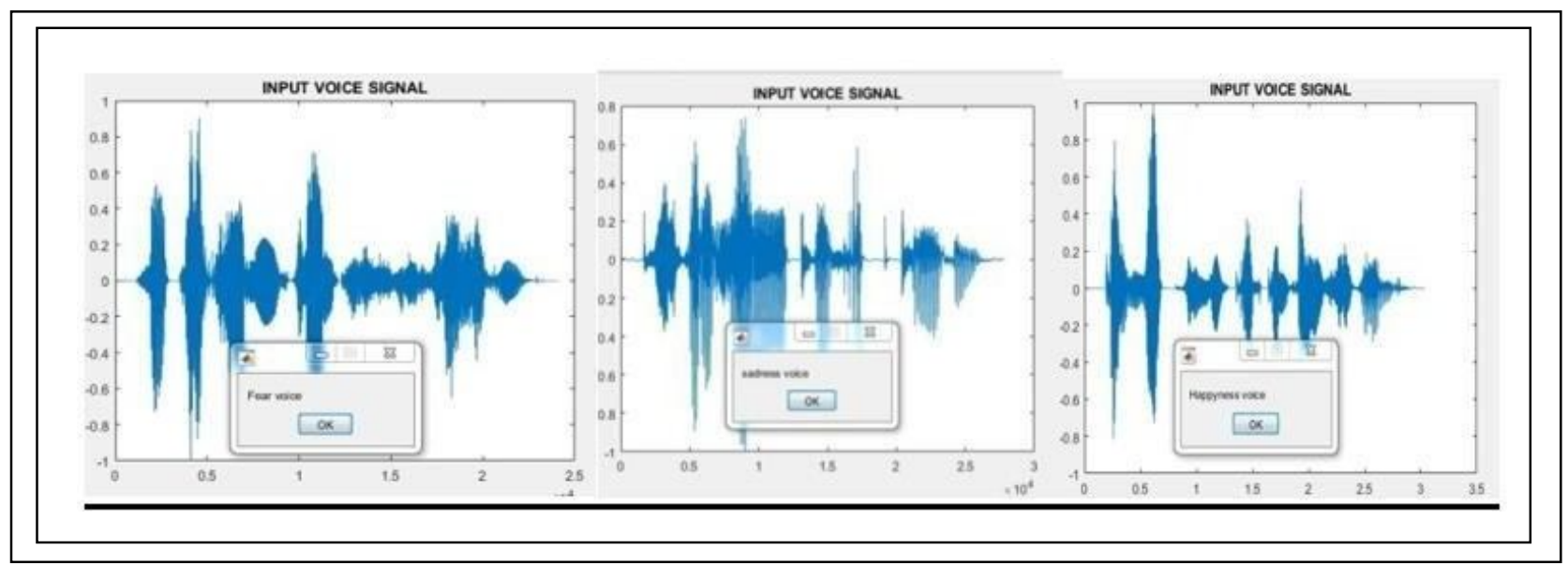

Figure 5: Speech Emotion Recognition using MFCC (Fear, Sadness, Happiness)

In any case, it's found the predictable values including pitch related values, formants features, essentialness features, and timing features pass on huge energetic prompts. There are in like manner TEO based algorithms proposed for recognizing neutral ways concentrated on talk. Notwithstanding the way that the recently referenced features are important for seeing unequivocal sentiments, there is no satisfactorily suitable component to portray tangled energetic states shows in Figure 4 and Figure 5.

\section{CONCLUSION}

In past examinations, various highlights were utilized for discourse feeling acknowledgment. Right now, proposed MFCC, PLP and LPC model to separate striking acknowledgment by consolidating MFCC with PLP and LPC highlights. These outcomes set up that the proposed model is useful for speaker- autonomous discourse feeling acknowledgment. In future, with additional time and with progressively far reaching research the proposed framework can be made increasingly exact. Additionally, new traffic sign location calculations can be added in order to give a more extensive assortment of alternatives to browse.

\section{REFERENCES}

[1] S. R. Bandela and T. K. Kumar, "Emotion Recognition of Stressed Speech Using Teager Energy and Linear Prediction Features," 2018 IEEE 18th International Conference on Advanced Learning Technologies (ICALT), Mumbai, 2018, pp. 422-425. 
[2] S. Ramamohan and S. Dandapat, "Sinusoidal modelbased analysis and classification of stressed speech," in IEEE Transactions on Audio, Speech, and Language Processing, vol. 14, no. 3, pp. 737-746, May 2006.

https://doi.org/10.1109/TSA.2005.858071

[3] A. Mencattini, E. Martinelli, F.Ringeval,B. Schuller and C. D. Natale, "Continuous Estimation of Emotions in Speech by Dynamic Cooperative Speaker Models," in IEEE Transactions on Affective Computing, vol. 8, no. 3, pp. 314-327, 1July-Sept. 2017.

[4] P. Shih, C. Chen and C. Wu, "Speech emotion recognition with ensemble learning methods," 2017 IEEE International Conference on Acoustics, Speech and Signal Processing (ICASSP), New Orleans, LA, 2017, pp.2756-2760.

[5] Y. Sun, Y. Zhou, Q. Zhao and Y. Yan, "Acoustic Feature Optimization for Emotion Affected Speech Recognition," 2009 International Conference on Information Engineering and Computer Science, Wuhan, 2009, pp.1-4.

[6] R. Rajak and R. Mall, "Emotion recognition from audio, dimensional and discrete categorization using CNNs," TENCON 2019 - 2019 IEEE Region 10 Conference (TENCON), Kochi, India, 2019, pp. 301305.

https://doi.org/10.1109/TENCON.2019.8929459

[7] N. Kamaruddin, A. W. Abdul Rahman and N. S. Abdullah, "Speech emotion identification analysis based on different spectral feature extraction methods," The 5th International Conference on Information and Communication Technology.

[8] S. Pathak and A. Kulkarni, "Recognizing emotions from speech," 2011 3rd International Conference on Electronics Computer Technology, Kanyakumari, 2011, pp. 107-109

[9] Syamala, M., \& Nalini, N. J. (2019). A Deep Analysis on Aspect based Sentiment Text Classification Approaches. International Journal of Advanced Trends in Computer Science and Engineering (IJATCSE), 8(5), 1795-1801.

https://doi.org/10.30534/ijatcse/2019/01852019

[10] Putra, R. R., Johan, M. E., \&Kaburuan, E. R. (1856). A Naïve Bayes Sentiment Analysis for Fintech Mobile Application User Review in Indonesia. International Journal of Advanced Trends in Computer Science and Engineering, 1860.

https://doi.org/10.30534/ijatcse/2019/07852019

[11] Teja, M. S., Reddy, M. R., \& Aishwarya, R. (2020, May). Man-on-Man Brutality Identification on Video data using Haar Cascade Algorithm. In 2020 4th International Conference on Intelligent Computing and Control Systems (ICICCS) (pp. 274-278). IEEE. 